\title{
Preparation and Characterization of Silver Nanospheroids: Theoretical and Experimental Approaches
}

\author{
Mounira Amraoui, Chouaib Daoudi, ${ }^{*}$ and Mohamed Remram \\ Laboratoire LEMEAMED, Faculté des Sciences de la Technologie, Université des Frères Mentouri Constantine1, \\ Constantine 25000 Algeria
}

Received November 13, 2016; accepted March 08, 2017; published June 30, 2017

\begin{abstract}
We synthesized silver nanoparticles of different sizes and forms as a function of the molar masses of $\mathrm{AgNO}_{3}$, in two different solvent mediums (ethanol, methanol). We carried out the synthesis according to standard chemical route with major modifications. The spectroscopic characterization showed the presence of two distinct absorption bands; the first band at 300nm and the second shifted to $440 \mathrm{~nm}$. We found a multipolar phenomenon depending on temperature. The shape and size predicted by the numerical model conform to the experimental results. The study demonstrated that the nanoparticles have a spheroidal shape.
\end{abstract}

Silver nanoparticles are particularly attractive due to their unique optical properties. For example, they exhibit a strong absorption band in the UV-visible region [1] Noble metals nanoparticles are used in many applications such as chemical sensors, biological sensors and imaging as well as the targeted photothermal treatment of various diseases such as cancer [2-3].

In addition, metal nanoparticles can be prepared with high accuracy in a range of well-defined structures like spheres, rods and wires by various methods, physical or chemical [4]. The studies have shown that the size and shape as well as physical and chemical properties of metal nanoparticles are strongly influenced by experimental conditions. The study of the synthesis of these nanoparticles as a function of reducing agents, stabilizers and physical conditions such as temperature is very interesting [5-7]. Therefore, the modeling of a synthetic process for controlling these parameters is one of major interests of ours.

The first objective of this work focuses on the synthesis of silver nanoparticles by chemical reduction of the $\mathrm{AgNO}_{3}$ precursor, for different molar masses, in two different solvents (ethanol and methanol). The second is the evaluation of the size and shape of the synthesized nanoparticles by a theoretical approach based on numerical analysis, depending on the refractive index of the obtained colloidal solution.

First, we used 0.0025 mole of $\mathrm{AgNO}_{3}$, mixed with $10 \mathrm{ml}$ of ethanol. The mixture was then added to a solution of benzoic acid $(0.5 \mathrm{~g}$ in $10 \mathrm{ml}$ of ethanol), and to a PVP

*E-mail: daoudi.chouaib@gmail.com solution ( $1 \mathrm{~g}$ in $10 \mathrm{ml}$ of ethanol). The resulting solution is vigorously stirred with a magnetic stirrer at room temperature for 20 minutes. Next, the solution is heated to different levels of temperature $\left(40^{\circ} \mathrm{C}, 50^{\circ} \mathrm{C}, 60^{\circ} \mathrm{C}\right.$, and $\left.70^{\circ} \mathrm{C}\right)$. After that, different samples are collected in test tubes and labeled accordingly.

A second set of samples was prepared by replacing ethanol with methanol, under the same conditions for each elaboration step. The color of samples gradually changes from yellow, dark yellow to reddish brown. Then, we varied the quantities of $\mathrm{AgNO}_{3}(0.0015,0.0025,0.0030$, 0.0035 mole) while keeping the other parameters constant.

The UV-Vis spectra of silver nanoparticles were recorded at room temperature using an Ultrospec 2100 PRO spectrophotometer with a standard quartz cuvette of $10 \mathrm{~mm}$ optical path.

We have characterized our colloidal solutions at 0.0025 mole of $\mathrm{AgNO}_{3}$, collected at different temperatures in the two solvents. In all obtained results, whatever the parameters used (see Figs. $1 \div 2$ ), we find the appearance of two distinct absorption bands; the first band appears at low wavelengths around $300 \mathrm{~nm}$ whereas the second is shifted to longer wavelengths around $440 \mathrm{~nm}$. These two absorption bands (300nm and 440nm) are certainly due to the presence of two symmetry axes ( $a$ and $b$ ) and the aspect ratio $\mathrm{a} / \mathrm{b}$ (see theoretical model below); therefore, we think that the synthesized nanoparticles are mostly nanorods or spheroids.

Figure1 shows the absorption band toward 440nm for silver nanoparticles in both solutions. Noting that in ethanol solvent, when the temperature increases, the absorption band intensity increases as well and without any variation in the peak positions; further, the full width at half maximum (FWHM) is still somewhat narrow. However, in a methanol solvent, the intensity of the absorption peak grows randomly, with respect to a temperature rise; although the absorption peak shifts to the right towards higher values, yet the appearance of another absorption band is noticed. In addition, we found that the positions of the absorption bands depend linearly on the dielectric constant of the medium; indeed, for both methanol and ethanol solutions, the measured refractive 
indices are 1.37 and 1.34 , respectively, the position of absorption bands registered for methanol is shifted compared to the ethanol solution.
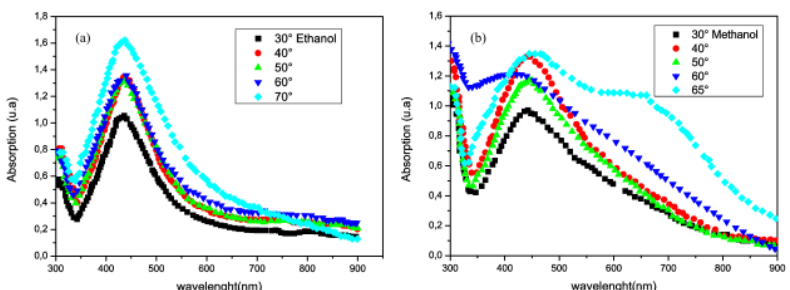

Fig. 1. UV-VIS absorption spectra of silver nanoparticles prepared in various solvents, (a) Ethanol, (b) Methanol, at a constant molar concentration of 0.0025 mole.

These results provide information about the presence of an agglomeration phenomenon, and clusters creation particularly in a methanol medium. In addition, beyond $60^{\circ} \mathrm{C}$, methanol reaches its boiling point and easily evaporates thus reducing its solvation process. Indeed, the presence of a PVP stabilizer during the chemical reduction process is to avoid precipitation and agglomeration of nanoparticles. However, we found that precipitation is limited with an ethanol solvent. On the other hand, with the methanol solvent, the absorbance peak is decreased and red-shifted. This phenomenon of agglomeration and precipitation of nanoparticles in different mediums has been observed by several authors [8-9].
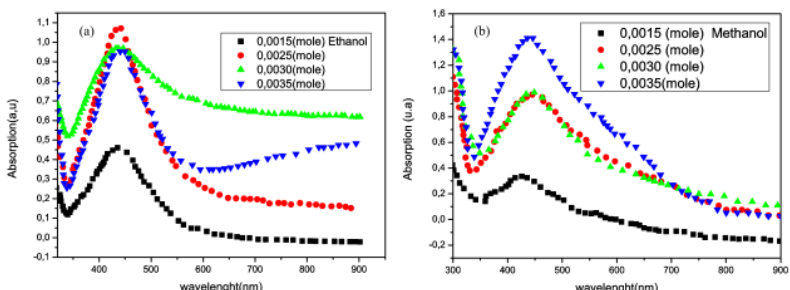

Fig. 2. UV-VIS absorption spectra of silver nanoparticles prepared in various solvents, (a) Ethanol, (b) Methanol, for a constant temperature of $30^{\circ} \mathrm{C}$.

Then, we changed the molar concentration of $\mathrm{AgNO}_{3}$ from 0.0015 mole to 0.0035 mole under the same conditions at ambient temperature. The best results are obtained at a concentration of 0.0025 mol. Better absorption peaks are noted and the spectrums less spread, in contrast with the other concentrations (see Fig. 2).

Elsewhere it is reported that the significant broadening of the resonance band, and the increase in the FWHM, of the corresponding peaks; (Fig. 2b), are the consequence of an increase in the size of the nanoparticles [10]. This increase of the nanoparticles can be explained by the agglomeration phenomenon, present in a methanol medium. Moreover, several authors have reported [9][11] that during the precipitation of two or more nanoparticles, the resulting absorption peak corresponds to the total volume of the new structure of the nanoparticles, which in most cases form a spheroidal shape with the corresponding volume and aspect ratio of initial nanoparticles.

The optical modeling of silver nanoparticles has been performed by the finite element method (FEM) [12], in which the variation in the absorption cross section is calculated with respect to the refractive index of a surrounding medium. The latter is ethanol $\left(n_{m}=1.34\right)$ and methanol $\left(n_{m}=1.37\right)$, respectively. The silver nanoparticle is modeled by $3 \mathrm{D}$ simulation using the commercial software COMSOL Multiphysics [13]. The electric field $\overrightarrow{\mathrm{E}}$ is calculated using the Helmholtz equation [14]:

$$
\nabla \times\left(\frac{1}{\mu_{r}} \nabla \times \overrightarrow{\mathrm{E}}\right)-k_{0}^{2}\left(\varepsilon_{r}-j \frac{\sigma}{\omega \varepsilon_{0}}\right) \overrightarrow{\mathrm{E}}=0,
$$

where $\varepsilon_{r}$ is the complex relative permittivity, $\mu_{r}$ is the relative permeability, $k_{0}$ is the wave number in vacuum and $\omega$ is the angular frequency. The absorbed energy by silver nanoparticles is obtained as follows [12]:

$$
Q_{a b s}=\frac{1}{2} \mathfrak{R}\left\lceil\iint_{s} \overrightarrow{\mathrm{E}}_{\mathrm{tot}} \times \overrightarrow{\mathrm{H}}_{\mathrm{tot}} \cdot \overrightarrow{\mathrm{n}} d s\right],
$$

where $\vec{E}_{t o t}$ and $\vec{H}_{t o t}$ represent the total electric and magnetic field vectors, respectively, $\mathrm{n}$ is the normal unit vector to the surface of the nanoparticle. The absorption cross section $\sigma_{a b s}$ is defined as following: $\sigma_{a b s}=Q_{a b s} / I_{0}$ where, $I_{0}=(1 / 2) \varepsilon_{0} n_{m} E_{0}^{2}$ is the intensity of the incident laser beam, $E_{0}$ represents the amplitude of the incident laser beam, $\varepsilon_{0}$ is the permittivity of vacuum and $n_{m}$ is the refractive index of a surrounding medium.In order to predict the shape of silver nanoparticles in our solution, we considered three geometrical models: spherical, rod and spheroid (Fig. 3) for different diameters ranging from $\mathrm{D}=8 \mathrm{~nm}$ to $12 \mathrm{~nm}$ and an aspect ratio between 1.2 and 1.5. The sizes of the silver nanoparticles are estimated from transmission electron microscopy results with correlation to imageJ particles analysis function [15]. The permittivity values of silver nanoparticles are extracted from Palik experimental data [16]. The nanoparticles are supposed to be immersed in a spherical environment surrounded by perfectly matched layers (PML); we have applied scattering boundary conditions on the external surfaces to consider an infinite domain. The nanoparticle is excited by an electromagnetic wave $400 \mathrm{~nm}$ to $800 \mathrm{~nm}$ long, polarized parallel to the major axis in the case of the spheroid and the rod. 


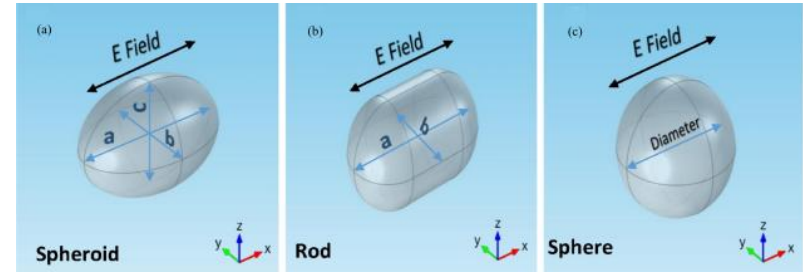

Fig. 3. Different shapes of nanoparticles modelled by COMSOL, (a) Spheroid, (b) Rod, (c) Sphere.

Typically, we assumed a diameter of $12 \mathrm{~nm}$ for the three types of nanoparticles and then varied their lengths between $8 \mathrm{~nm}$ to $10 \mathrm{~nm}$ for the spheroid and the rod, which corresponds to the aspect ratio of 1.2 to 1.5 .
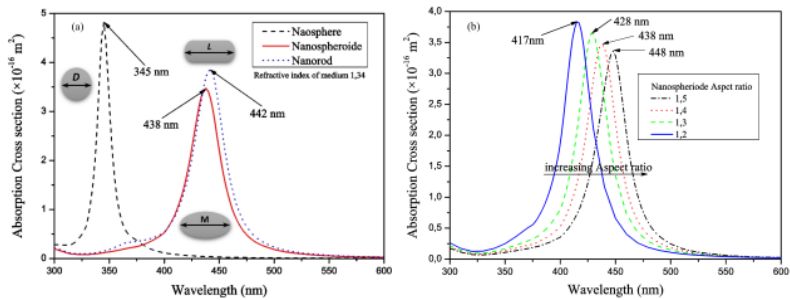

Fig. 4. Absorption cross section spectra calculated for silver nanoparticles immersed in Ethanol: (a) nanospheroid, nanorod and nanosphere $(\mathrm{D}, \mathrm{L}, \mathrm{M}=12 \mathrm{~nm}$ with fixed minor axes of $8.5 \mathrm{~nm}$ for the rod and spheroid respectively), (b) silver nanospheriod with aspect ratio varied from 1.2 to 1.5

Figure 4a shows the comparison of calculated absorption cross section for the three models with a fixed diameter, length and major axes $\mathrm{D}, \mathrm{L}, \mathrm{M}=12 \mathrm{~nm}$ and fixed minor axes of $8.5 \mathrm{~nm}$. The optimization of the absorption cross section for a spheroid nanoparticle is illustrated in Fig. 4b. The best results are obtained for the nanospheroid, especially for the geometrical parameter of ( $\mathrm{M}=12 \mathrm{~nm}$ and minor axes of $8.5 \mathrm{~nm}$ ). The numerical results are in agreement with the experimental results, especially for those forms and dimensions. Indeed, Fig. 5 shows the correlation between the calculation and experimental spectra for the solution of silver nanoparticles with 0.0025 mole in the ethanol solvent at a temperature of $30^{\circ} \mathrm{C}$.

Besides, the empirical spectra are broader than the simulated. Further, in the numerical calculation, we considered that the nanoparticles are isolated and homogeneous with regular shapes, on the other hand, for the experimental characterization, the nanoparticles in the colloidal solution have a random distribution, with complex forms due to the precipitations, agglomerations and different orientations to the incoming light. These parameters play an important role in the broadening and enhancing of experimental spectra, which explains the difference between the two spectra.

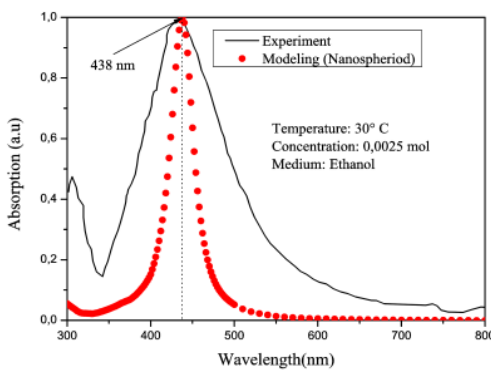

Fig. 5. Comparison between experimental and theoretical results for silver nanospheroid with $\mathrm{L}=12 \mathrm{~nm}$ and minor axes of $8.5 \mathrm{~nm}$.

In summary, we have synthesized silver nanoparticles by chemical route, for different molar masses of $\mathrm{AgNO}_{3}$ (0.0015 mole to 0.0035 mole) in two different solvents (ethanol and methanol). The UV-VIS absorption showed the emergence of two distinct absorption bands one at 300 $\mathrm{nm}$ and the other at $440 \mathrm{~nm}$. This proves the existence of heterogeneous forms in the solution, which is confirmed by the theoretical model. We asserted that the synthesized silver nanoparticles are of spheroidal shape and about 12 $\mathrm{nm}$ size. Furthermore, we found in the methanol medium, a significant broadening of the resonance band, and important values of FWHM, of the corresponding peaks; the consequences of increasing the size of nanoparticles and the appearance of the agglomeration phenomenon. The best numerical results in agreement with the experimental results are obtained for a nanospheroid with the 1.4 aspect ratio.

\section{References}

[1] C. Noguez, J. Phys. Chem. C 111(10), 3806 (2007).

[2] M. Amraoui, C. Daoudi, M. Remram, Int. Sc. Sci and Tech. Conf. 2015 Aydın, Turkey, 11-13 May 2015 (ISSTC 2015).

[3] M.I. Gonzalez-Sanchez et al., Mater. Sci. Eng C. Mater. Biol. Appl 50, 332 (2015).

[4] X. Dong et al., J. Phys. Chem. C 113(16), 6573 (2009)

[5] A. Wolak, M. Grabiec, O. Véron, J.-P. Blondeau, K. Dzierżęga, Phot Lett. Poland 5(2), 54 (2013).

[6] A. Zielinska, E. Skwarek, A. Zaleska, M. Gazda, J. Hupka, Procedia Chemistry 1, 1560 (2009).

[7] K-S. Chou, Y. Chang, L. Hua, Ind. Eng. Chem. Res 51(13), 4905 (2012).

[8] A.M. Atta, H.A. Al-Lohedan, A.O. Ezzat, Molecules 19(5), 6737 (2014)

[9] S. Botasini, E. Méndez, J. Nanopart. Res. 15, 1526 (2013).

[10] V. Amendola, O.M. Bakr, F. Stellacci, Plasmonics 5(1) 85 (2010).

[11] L. Jeong, W. Ho Park Int. J. Mol. Sci 15, 6857 (2014).

[12] Peter Monk, Finite element method for Maxwell's equations, (Oxford, Clarendon Press 2003).

[13] COMSOL Multyphisics, RF Module, User's guide (2008).

[14] C.F. Bohren, D.R. Huffman, Absorption and Scattering of Light by Small Particles (New York, A Wiley-Interscience Publication 1998).

[15] M.D. Abramoff, P.J. Magalhaes, S.J. Ram, Biophotonics International 11(7), 36 (2004)

[16] E.D. Palik, Handbook of optical constants of solids (California, Academic Press Inc, 1985). 